\title{
ArcheoSciences
}

Revue d'archéométrie

\section{Les pierres dressées de la forêt du Gâvre (Loire- Atlantique) : nature et origine des matériaux, premiers éléments de chronologie (14C, OSL)}

Serge Cassen, Sophie Blain, Pierre Guibert, Guirec Querré et Cyrille Chaigneau

\section{(2) OpenEdition}

Édition électronique

URL : http://journals.openedition.org/archeosciences/4057

DOI : $10.4000 /$ archeosciences.4057

ISBN : 978-2-7535-2755-3

ISSN : $2104-3728$

\section{Éditeur}

Presses universitaires de Rennes

Édition imprimée

Date de publication : 17 avril 2013

Pagination : 173-188

ISBN : 978-2-7535-2757-7

ISSN : $1960-1360$

\section{Référence électronique}

Serge Cassen, Sophie Blain, Pierre Guibert, Guirec Querré et Cyrille Chaigneau, «Les pierres dressées de la forêt du Gâvre (Loire-Atlantique) : nature et origine des matériaux, premiers éléments de chronologie (14C, OSL) ", ArcheoSciences [En ligne], 37 | 2013, mis en ligne le 17 avril 2015, consulté le 19 avril 2019. URL : http://journals.openedition.org/archeosciences/4057 ; DOI : 10.4000/ archeosciences.4057 


\title{
Les pierres dressées de la forêt du Gâvre (Loire-Atlantique) : nature et origine des matériaux, premiers éléments de chronologie $(\mathrm{C14}, \mathrm{OSL})$
}

\author{
Standing stones of Le Gâvre forest (Loire-Atlantique, France): \\ nature and origin of materials, preliminary dating results (C14, OSL)
}

\author{
Serge Cassen*, Sophie Blain**, Pierre Guibert**, \\ Guirec Querré***, Cyrille Chaigneau****
}

\begin{abstract}
Les ouvrages de pierres dressées sont une composante essentielle de l'étude générale des mégalithes de la Préhistoire récente. Mais la perduration du phénomène ouest-européen, sur près de trois millénaires, laisse supposer des nuances qui à l'heure actuelle sont peu perceptibles, faute d'observations suffisantes sur un objet architectural au surplus difficile à définir. Dans l'Ouest de la France, les "alignements de menhirs » en roche quartzitique forment un sous-ensemble original mal connu. Établir leur encadrement chronologique apparait comme une priorité. L'exercice a été entamé sur un site inédit en forêt du Gâvre (nord de Nantes) à l'aide de trois sondages restreints permettant d'accéder aux couches archéologiques au contact de trois monolithes. À titre expérimental, une datation OSL directe a été envisagée, mais les grains de quartz provenant des deux blocs et d'une pierre de calage également en quartz n’ont pas émis de signal de luminescence suffisant pour obtenir des données assez précises pour la datation. Une alternative a néanmoins été proposée en datant le dépôt des sédiments du comblement de la fosse du monolithe N15. L'intervalle de dates [2040-1680] av. J.-C. (1 $\sigma)$ situe le creusement de la fosse et donc l'érection de la stèle dans le Bronze ancien. Cette datation est en accord avec celle obtenue pour le second sondage N6 A [1752-1628] av. J.-C. (2 $\sigma)$ grâce à l'analyse ${ }^{14} \mathrm{C}$ d'un charbon de bois de la fosse d'implantation.

Stone rows are a major component of the study of late Prehistoric megaliths. The western phenomenon which lasted for about three millennia is no yet well defined due to the lack of sufficient studies on this architectural object. In western France, the rows of menhirs in quartzitic rock constitute a not well known but overall original subset. Assessing their chronology is therefore a priority. In this context, the unpublished megalith site of the Pilier in the northern area of the forest of Le Gavre (north of Nantes, France) was selected. Three limited excavations allowed recording the archaeological layers in contact with three monoliths. As an experimental test, a direct OSL dating was considered, but the grains of quartz coming from two blocks and from a shim stone, were very dim making them unsuitable for dating purposes. An alternative was nevertheless offered by dating the sediments filling the pit of the monolith N15. The OSL date range: [2040-1680] BC (1 $\mathrm{\sigma})$ places the digging of the pit and therefore the erection of the stele in the early Bronze Age. This chronological interval is in agreement with the ${ }^{14} \mathrm{C}$ date obtained from a charcoal from the pit foundation of a second stone N6A [1752-1628] BC (2 $\left.\sigma\right)$.
\end{abstract}

Mots-clés: chronologie, datation par luminescence, pétrographie, pierres dressées, radiocarbone.

Keywords: chronology, luminescence dating, petrography, radiocarbon, standing stones.

* CNRS, Laboratoire de recherches archéologiques (UMR 6566, Centre de Recherche en Archéologie, Archéosciences, Histoire), Université de Nantes, BP 81227, 44312 Nantes cedex 3, (Serge.Cassen@univ-nantes.fr).

** CNRS, Iramat-CRP2A, (UMR5060, Institut de Recherche sur les Archéomatériaux-Centre de Recherche en Physique Appliquée à l'Archéologie), Université de Bordeaux 3, Maison de l'Archéologie, Esplanade des Antilles, 33607 Pessac cedex, (blain.sophie@gmail.com; guibert@u-bordeaux3.fr).

*** Laboratoire ArchéoSciences (UMR 6566, Centre de Recherche en Archéologie, Archéosciences, Histoire), Université de Rennes 1 - Campus de Beaulieu, 35042 Rennes cedex, (guirec.querre@univ-rennes1.fr).

**** Musée de Carnac, 10 place de la Chapelle, 56340 Carnac, (c.chaigneau@museedecarnac.fr). 


\section{INTRODUCTION, PROBLÉMATIQUE}

Lors de la mise en évidence de «l'alignement du Grand Menhir ", en 1989, sur la commune de Locmariaquer en Morbihan (Cassen, L'Helgouac'h, 1992; L'Helgouac'h, Cassen, 2009; Cassen, 2009b), ce type architectural fait de pierres dressées, jusqu'ici assez énigmatique, fut instantanément rapproché de l'autre phénomène, chronologiquement postérieur, relatif au réemploi des stèles dans la construction des tombes à couloir (Minot, 1964; Jubert, 1974; L'Helgouac'h, 1983) datées en règle générale de la première moitié du IV ${ }^{e}$ millénaire av. J.-C. La problématique des ouvrages de stèles (files rectilignes ou curvilignes) était à cette occasion relancée, et fit l'objet de différentes approches quant à leur détection, leur description et leur datation, notamment en région de Carnac (Cassen et al., 2009).

"L'alignement de menhirs" est cependant un appellatif insuffisant qui recouvre au surplus bien des variantes architecturales. Nous en sommes d'ailleurs à un stade de déchiffrement comparable à celui qui révélait la multitude des "dolmens" à la fin du XIX siècle, et qui se donnait pour tâche prioritaire d'ordonner leurs plans par le biais de typologies. L'expérience acquise et le savoir cumulé sur ces tombes à couloir et ces tombes à vestibule ne trouvent donc pas d'équivalent à ce jour pour éclairer ce que nous nommons "barres de stèles ", dont certaines sont datées (relations stratigraphiques, mobilier, dates ${ }^{14} \mathrm{C}$ ) du milieu $\mathrm{du} \mathrm{V}^{\mathrm{e}}$ millénaire, à l'image justement du Grand Menhir.

Parmi les dizaines de sites inventoriés dans l'Ouest, qui semblent ne pas dépasser le Massif armoricain sinon par résurgences sur des territoires très spécifiques liés au Morbihan par le jeu des échanges compétitifs et de la "religion» au sens large (Bourgogne, Suisse - Cassen, à paraître), un sous-ensemble original se distingue par le matériau (quartz, grès) et par son implantation plutôt réservée à la partie orientale de la Bretagne, jusqu'en Anjou (Gruet, 1967). Dans le cadre d'un modèle interprétatif, et notamment à travers plusieurs tests prédictifs, nous nous sommes interrogés sur les rapports historiques entre ces ouvrages en roche quartzitique et ceux plus classiques en roches métamorphiques (schistes, gneiss, granites) implantés aux abords du littoral sud-armoricain, en posant pour les premiers la possibilité d'une réalisation plus récente (Cassen, 2009a, p. 116). Une possibilité somme toute comparable à celle autrefois illustrée par les "allées couvertes", puisque leur distribution généralisée dans l'Ouest et leur positionnement topographique bien distincts des tombes à couloir, laissaient supposer une date différente de ces dernières.

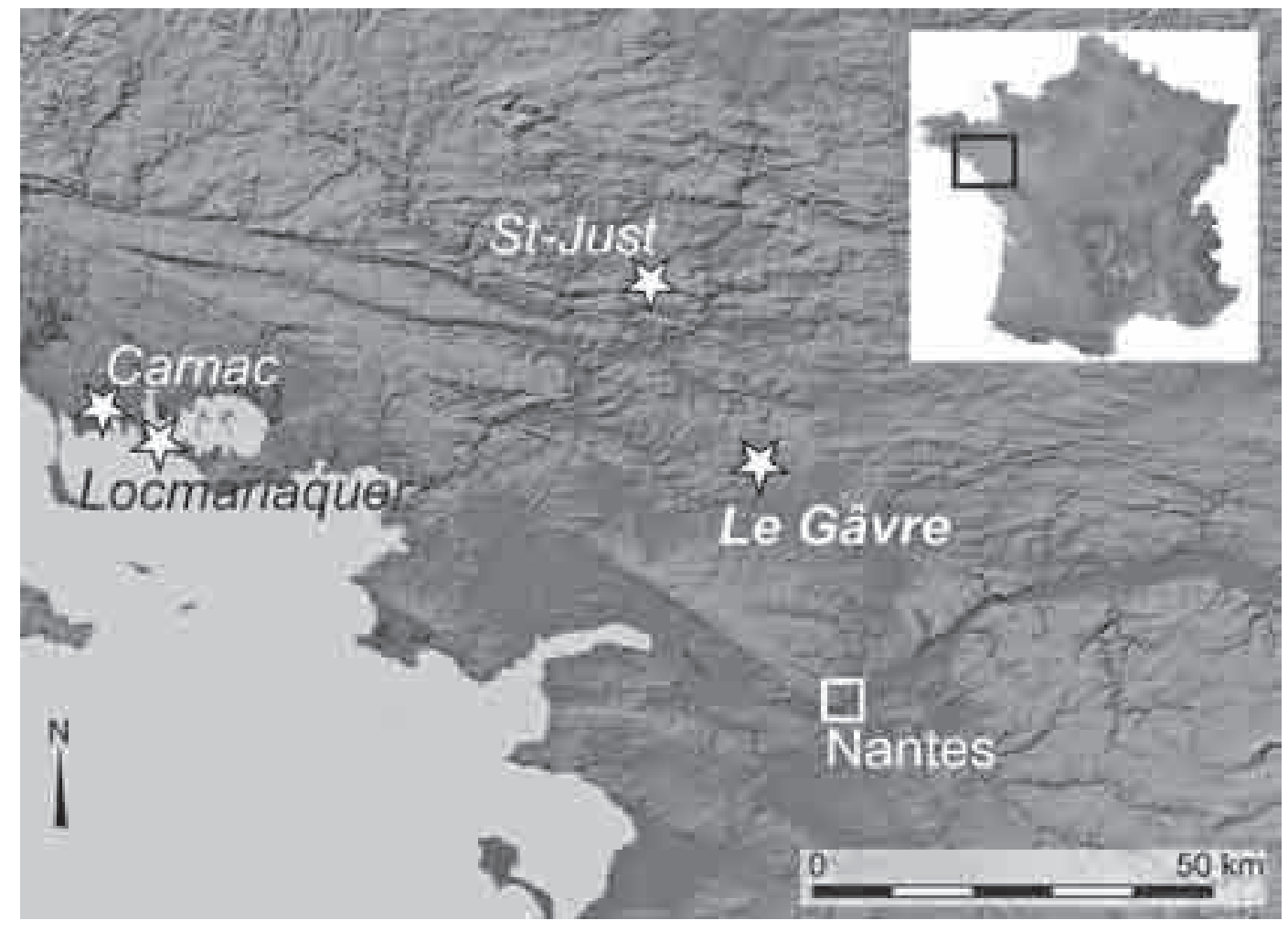

Fig. 1: Carte de situation des sites archéologiques à pierres dressées mentionnés dans le texte (Morbihan, Illeet-Vilaine, LoireAtlantique).

Fig. 1: Situation map of the standing stones archaeological places mentioned in the text (Morbihan, Ille-et-Vilaine, LoireAtlantique). 

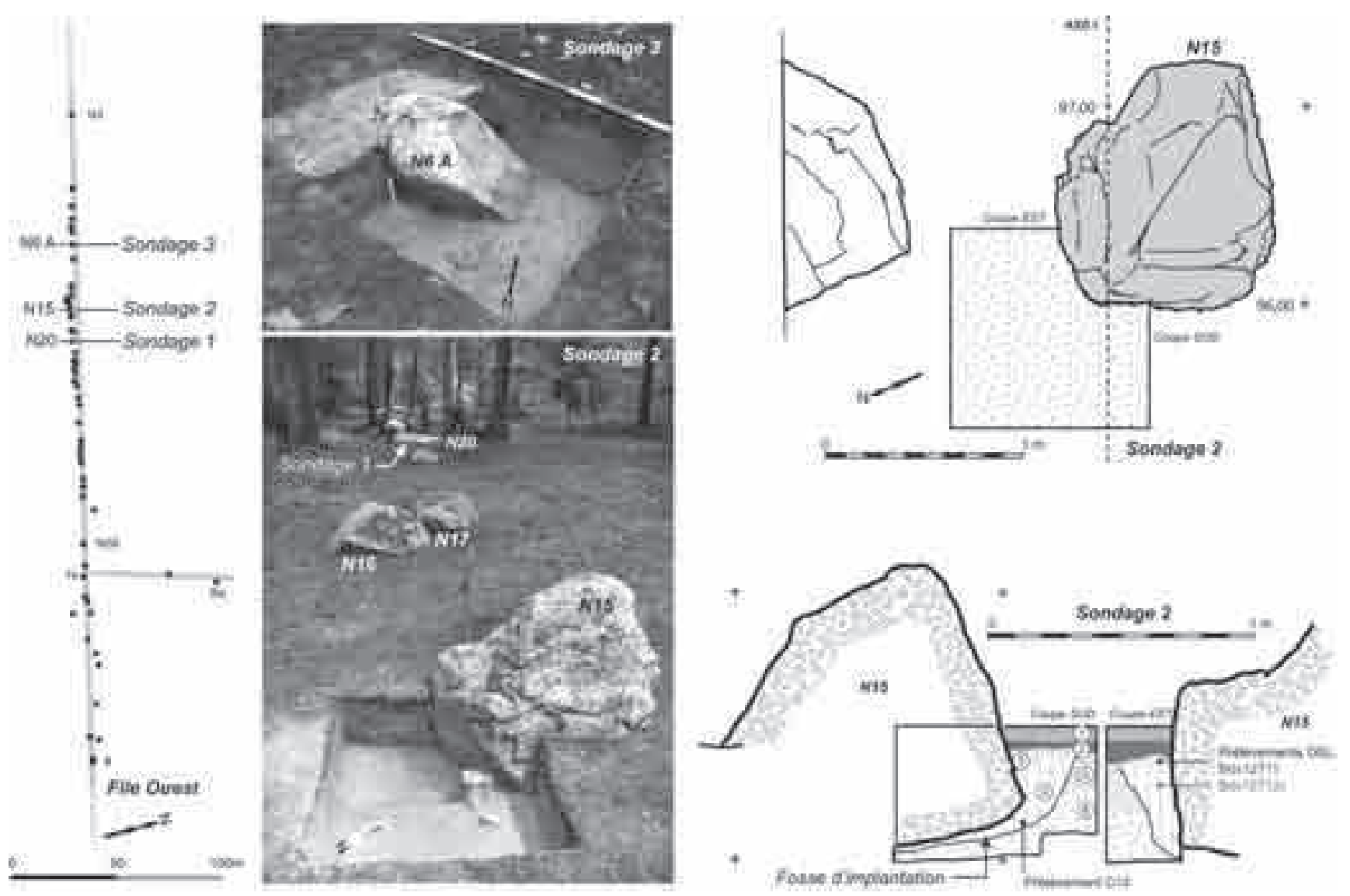

Fig. 2: (Voir planche couleur) Plan simplifié de la partie occidentale du site; emplacement des sondages archéologiques; localisation des prélèvements.

Fig. 2: (See colour plate) Simplified plan of the western part of the site; location of the archaeological excavations; localization of the samples.

Devant cette chronologie incertaine, la découverte de blocs alignés dans la forêt de Gâvre (Loire-Atlantique fig. 1) nous a semblé, en 2007, être l'occasion de tester ces propositions et tenter une datation, particulièrement en raison de leur éloignement de la région carnacoise dont la profusion des sites et le poids historique des aménagements ont tendance à brouiller la réflexion et la lecture des objets (Boujot, Pinet, 2007). Enfin, en milieu forestier difficile d'accès, les monolithes ont été jusqu'ici protégés des façons culturales, offrant de meilleures conditions de conservation.

L'ouvrage du Pilier se compose d'une première file occidentale allongée sur $400 \mathrm{~m}$, divisée plus à l'est en deux files parallèles de $500 \mathrm{~m}$ de long, sur l'ensemble desquelles s'ajoutent trois files secondaires perpendiculaires. Prospections et enregistrement au GPS ont précédé un levé topographique précis puis une campagne de trois sondages archéologiques restreints (2008) au contact de trois monolithes (fig. 2).
L'énormité du développement architectural imposait de rechercher des moyens de l'interroger rapidement, directement et peut-être même avec une moindre précision. C'est en cela que la méthode de datation par luminescence stimulée optiquement (OSL) et la réflexion sur une nouvelle technique de datation, telle que l'OSL de surface, nous ont semblé une manière d'entrer dans le problème posé par ces structures, en datant monolithes et sédiments au creux de leurs fosses d'implantation.

\section{NATURE DES ROCHES COMPOSANT L'ALIGNEMENT}

Les blocs de roche formant l'alignement du Pilier sont, en grande majorité, constitués de quartz blanc laiteux, ce qui est le cas pour l'un des 2 blocs étudiés par OSL (menhir N6A). Certains blocs, comme le second destiné à l'étude OSL (N15A), présentent des enclaves schisteuses de 


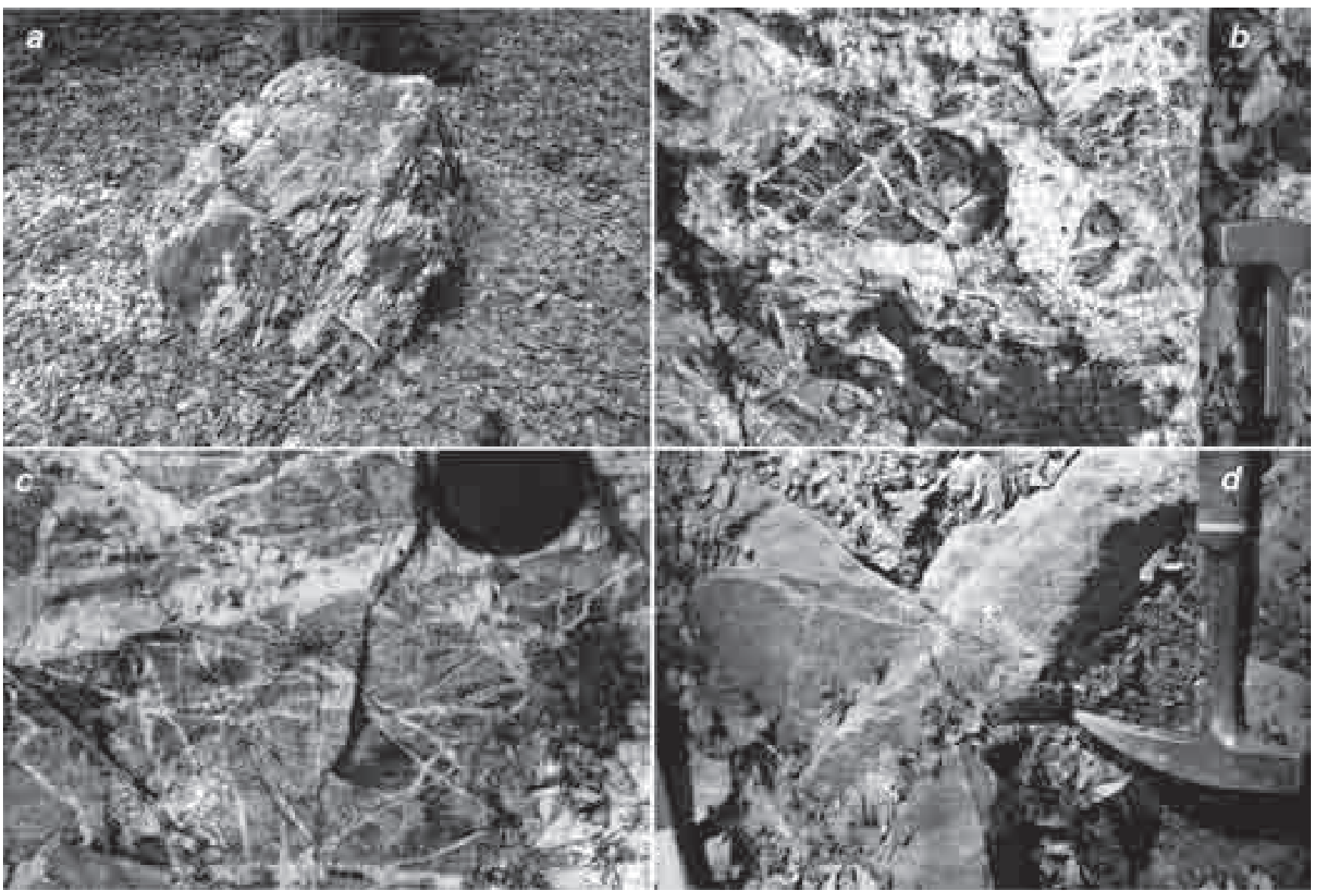

Fig. 3: (Voir planche couleur) Principaux faciès de la roche constituant les blocs de l'alignement et de l'encaissant.

Fig. 3: (See colour plate) Main facies of the stone constituting the blocks of the alignment and bed-rock.

a: Bloc montrant la forte structuration d'origine métamorphique de la roche. b: Présence d'enclaves mélanocrates dans la masse filonienne. Le quartz laiteux est prédominant par rapport aux enclaves. c: Vue rapprochée d'un bloc constitué de schiste encaissant parcouru de plusieurs filons de quartz laiteux (encaissant dominant). d: Schiste de l'encaissant.

a: Block showing the strong structuring of metamorphic origin of the rock. b: presence of melanocratic enclaves in the filonian mass. The milky quartz is dominant compared with the enclaves. c: view moved closer to a block constituted of schist browsed by several veins of milky quartz. d: schist of enclose bed-rock.

couleurs foncées, elles-mêmes souvent recoupées par de nombreux filonnets de quartz. Plus rarement, des blocs sont principalement composés de schistes gris-vert mais toujours zébrés de filons de quartz blanc (fig. 3).

Un petit fragment de roche détaché du bloc $n^{\circ} 20$ (système oriental, file sud), sans doute lors du passage d'un engin, a fait l'objet d'une étude en lame mince. La roche apparaît constituée de grandes plages de quartz en cristaux xénomorphes présentant des craquelures toutes dirigées dans le même sens, sans doute sous l'effet de contraintes tectoniques (fig. 4).

Il s'agit donc d'une roche magmatique de composition hyperquartzique. Cette roche est connue parfois sous le nom de silexite, terme qui porte cependant à confusion, trop proche des silex et silexoïdes, roches siliceuses également mais de nature sédimentaire. Il ne s'agit pas non plus d'un quartzite, terme réservé à une roche sédimentaire. L'appellation de quartzolite retenue ici et reconnu par la communauté internationale correspond à une roche ignée dont la proportion de quartz est supérieure à $90 \%$ (Streckeisen, 1974), ce qui est le cas des blocs de l'alignement du Gâvre.

Les roches composant l'alignement peuvent être parfois de couleur noire à rouge. Une analyse par PIXE de ces concrétions rouges à noires montre qu'il s'agit d'oxydes de fer qui par altération donnent une couleur rouge. Cette couleur 


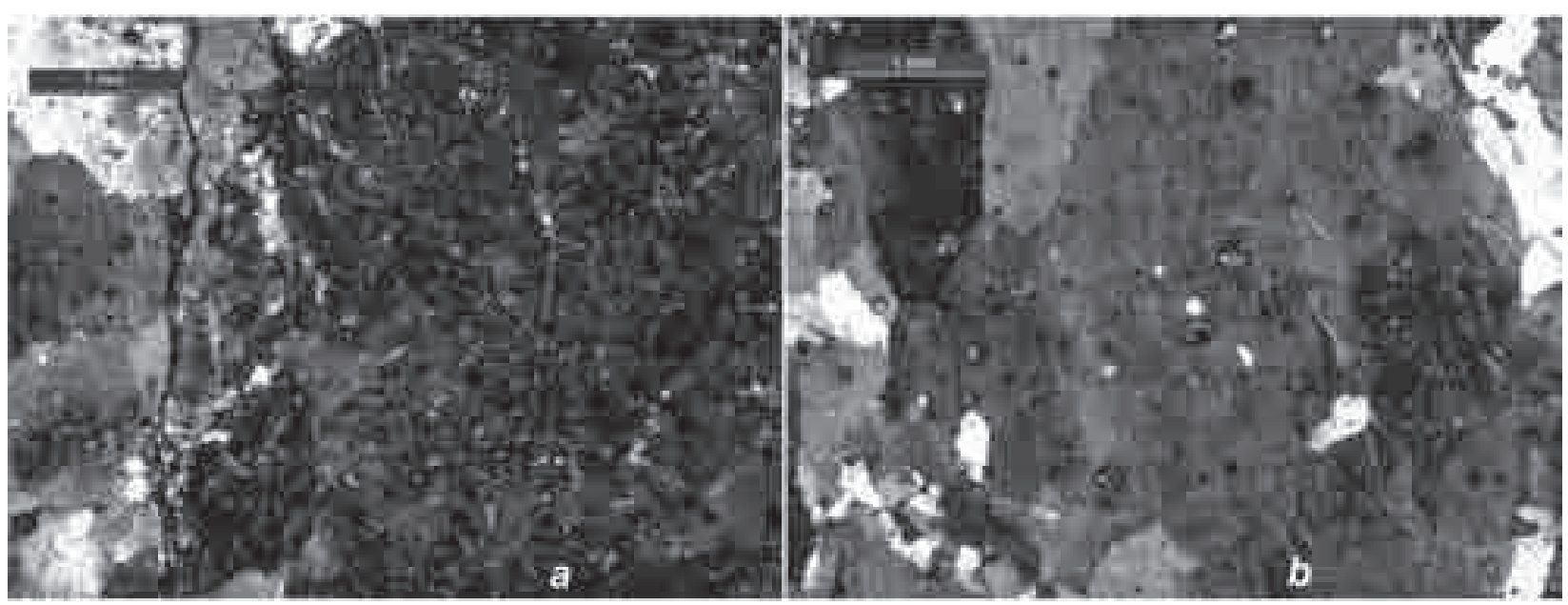

Fig. 4 (a et b) : Micrographies en lame mince de la roche constituant les blocs dressés du Pilier (polariseur et analyseur croisés, échelle linéaire $1 \mathrm{~mm})$.

Fig. 4 (a et b): Microwritten forms in thin blade of the rock constituting the Pilier stone row (polarizer and analyzer crossed, linear scale $1 \mathrm{~mm})$.

ne correspond donc pas à une éventuelle rubéfaction de la roche.

Les blocs de l'alignement sont constitués typiquement d'une roche filonienne essentiellement quartzeuse. Ce ou ces filons sont intrusifs dans des roches de type schistes et grès. Du point de vue géologique, l'alignement du Pilier se trouve dans la zone couverte par le synclinorium de SaintGeorges-sur-Loire. Si l'on fait abstraction des formations superficielles meubles attribuées au Plio-Pleistocène, le site se trouve sur un socle composé de roches sédimentaires datées de l'Ordovicien et du Silurien (cartes géologiques Nozay $n^{\circ} 420$ : Trautmann, 1988; et Redon n ${ }^{\circ} 419$ : Delfour et al., 1984). L'alignement se trouve plus précisément à l'interface des schistes de Saint-Perreux composés de siltites argileuses verdâtres à rougeâtres avec des intercalations de phtanites, d'âge ordovicien, et des grès de l'Eclys, grès séricitiques recristallisés, plutôt d'âge silurien. De plus, apparaissent sur les cartes géologiques de nombreux filons de quartz dont certains minéralisés en or ou/et pyrite. L'observation sur les blocs du site archéologique est identique à la description qui en est faite dans la légende des cartes, y compris la présence d'enclaves schisteuses chloritisées.

La carte de Nozay ne figure pas précisément la présence d'un filon de quartz à l'emplacement de l'alignement du Pilier (Trautmann, 1988). Toutefois, il est noté dans la légende de la carte de Redon la présence de nombreux filons quartzeux mais « qu'il n'est pas possible de représenter sur la carte à $1 / 50000$ " (Delfour et al., 1984). Par contre, un filon de quartz orienté Nord $110^{\circ}$, anciennement exploité, est relevé parfaitement dans le prolongement nord-ouest de l'alignement à environ $1,5 \mathrm{~km}$, à l'interface des formations ordoviciennes et siluriennes, ce qui est également le cas de la position de l'ouvrage de pierres dressées sur la feuille de Nozay (fig. 5). Il est donc tout à fait possible que ce filon se prolongeait plus au sud-ouest et qu'il fut exploité par les constructeurs protohistoriques.

L'orientation de l'alignement du Pilier est effectivement comprise entre l'azimut $106^{\circ}$ (file ouest) et l'azimut $112^{\circ}$ (files est), semblable à la direction structurale des formations géologiques locales, dont le filon de quartz figurant sur la carte géologique (fig. 5). Selon toute vraisemblance et du fait de cette concordance des alignements géologiques et archéologiques, il est probable que les blocs de quartz furent prélevés à proximité immédiate de l'ouvrage projeté, pour les disposer sans transport significatif tout en conservant la direction naturelle du filon. L'érosion différentielle du socle entre, d'une part, les filons de quartz relativement résistant et, d'autre part, les schistes souvent très altérés, a pu laisser en relief les roches filoniennes facilitant sinon leur accès au moins leur détection grâce à la topographie.

\section{IMPLANTATION DES SONDAGES ARCHÉOLOGIQUES}

Trois configurations distinctes sur le terrain furent testées dans les trois sondages restreints réalisés dans la partie occidentale de l'ouvrage (fig. 2). 


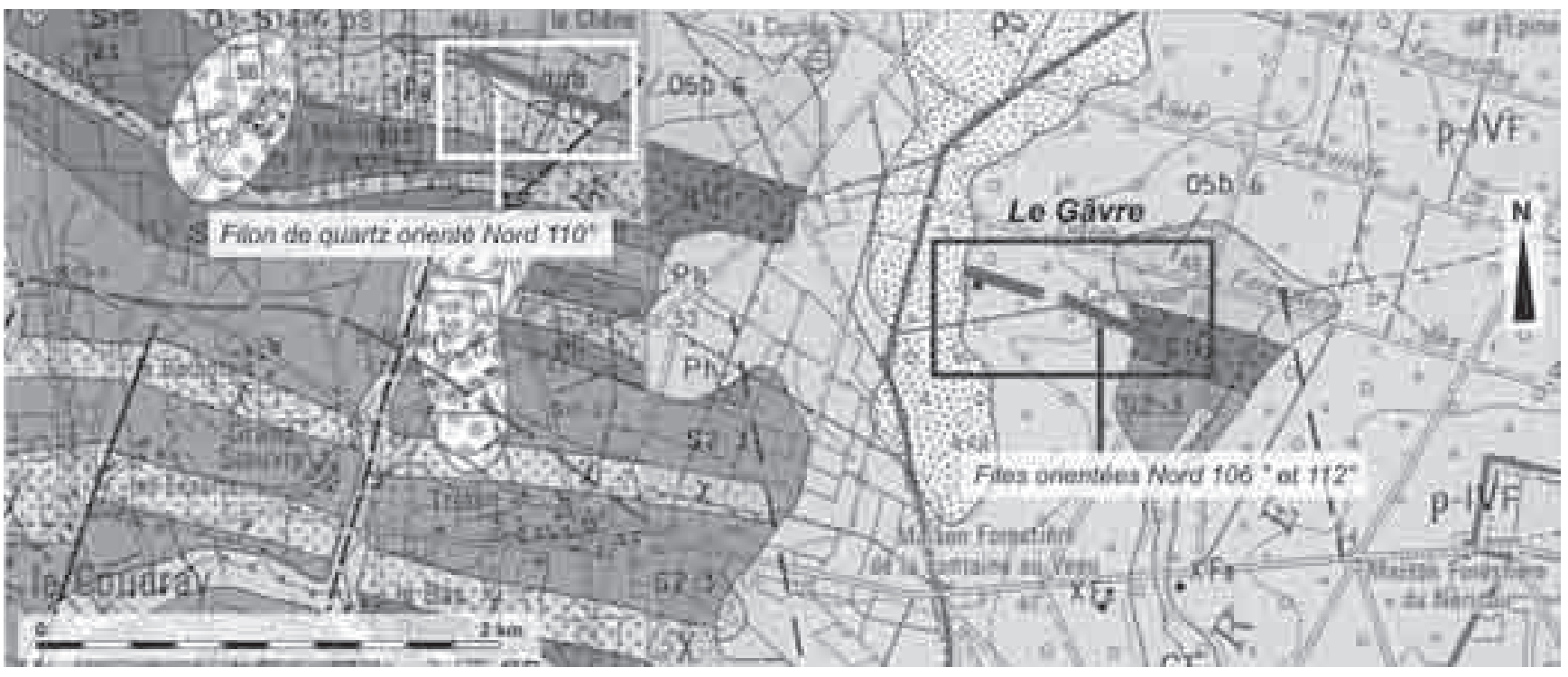

Fig. 5: (Voir planche couleur) Position de l'ouvrage du Pilier (Le Gâvre) replacé dans son contexte géologique.

Fig. 5: (See colour plate) Position of Pilier stone row (Le Gâvre) replaced in its geologic context.

La flèche symbolise l'orientation générale de l'alignement. Le filon de quartz se trouve à $1,5 \mathrm{~km}$ au NO de l'extrémité ouest de cet ouvrage, entre le Chêne et le Breil (zone notée Q et qua pour la carrière) (carte géologique BRGM Nozay n ${ }^{\circ} 420$ ).

The arrow symbolizes the general orientation of the alignment. The vein of quartz is $1,5 \mathrm{~km}$ in western $N O$ of the extremity of this site, between le Chêne and le Breil (zone noted $Q$ and qua for the quarry) (Geological map BRGM Nozay $n^{\circ} 420$ ).

Le sondage $\mathrm{N}^{\circ} 1$ a été ouvert à l'emplacement du bloc N20. Il s'agit d'une dalle horizontale, manifestement chutée; aucune fosse d'implantation n'a été détectée dans le quadrant (1 m x $1 \mathrm{~m}$ ) ouvert au contact nord-est de la pierre. Une argile d'altération apparaît sous l'horizon humifere. Aucun prélèvement de roche n’a été réalisé sur la face enfouie du bloc.

Le sondage № 2 a été ouvert autour de la partie basale septentrionale du bloc N15. Ce choix a été dicté par la morphologie massive laissant supposer un maintien dans la position d'origine du monolithe. La fosse d'implantation est cette fois bien identifiée: sous l'horizon humifère (C1 et $\mathrm{C} 2$, fig. 2), son comblement est composé d'une première couche formée d'un mélange de limon brun et jaunâtre (C3), puis d'un limon fin jaunâtre clair (C4) dans lequel fut prélevé un charbon en vue d'une datation ${ }^{14} \mathrm{C}$. Les deux prélèvements de sédiments en vue de la datation OSL proviennent de C3 et C4. L'encaissant est constitué d'une première couche de limon jaunâtre (C5) superposée à un horizon d'altération argilo-limoneux jaune clair (C6) contenant de petits galets de quartz et des plaquettes de schiste (cf. fig. 1). Le substrat schisteux n'a pas été atteint.

Le sondage $\mathrm{N}^{\circ} 3$ a été réalisé à la base du bloc N6 A (fig. 1). Une fosse d'implantation au comblement unique bien différencié est discernable autour de la pierre dont la taille est très réduite, choisie d'ailleurs pour cette raison dans notre enquête afin de contraster la morphologie de N15: haute de $50 \mathrm{~cm}$, elle devait au mieux émerger de $30 \mathrm{~cm} \mathrm{du}$ sol avant son affaissement oblique dans le limon argileux. Quelques pierres quartzitiques éparses trouvées à son pourtour pourraient être interprétées comme un " calage ", mais ce diagnostic n'est pas plus assuré que pour les petits blocs trouvés précédemment à la base de N15, inutiles dans cette fonction supposée. Un charbon de bois a été prélevé pour datation ${ }^{14} \mathrm{C}$ dans le comblement anthropique; il n'a bien entendu que la valeur habituelle de ces restes carbonisés sans origine établie. Le substrat schisteux n'a pas été atteint.

\section{ÉTUDE CHRONOLOGIQUE}

\section{Datation par le radiocarbone}

Des charbons de bois ont été prélevés dans les trois sondages, dont deux seulement ont révélé les fosses de fondation mieux à même d'être datées. La série ${ }^{14} \mathrm{C}$ est limitée à deux échantillons provenant des fosses des blocs $\mathrm{N} 15$ et N6 A (soumis en 2008 au comité Artemis; résultats parvenus en 2010 - tableau 1). La détermination préalable des 


\begin{tabular}{|c|c|c|c|c|c|c|}
\hline$N^{\circ}$ monolithe & $\mathrm{N}^{\circ}$ sondage & Couche & Nature prélèvement & Code labo & $\hat{A} g e^{14} \mathrm{C} \mathrm{BP}$ & Âge av. J.-C. $(2 \sigma)$ \\
\hline N6A & Sondage 3 & 2 & $\begin{array}{l}\text { Charbon brindille noisetier }(8 \mathrm{~mm} \text {; } \\
\varnothing: 3 \mathrm{~mm})\end{array}$ & $\begin{array}{c}\text { Lyon6219 } \\
\text { (SacA-16633) }\end{array}$ & $3405 \pm 30$ & $1752-1628$ \\
\hline N15 & Sondage 2 & 4 & Charbon vieux chêne $(15 \mathrm{~mm})$ & Lyon6218 (SacA-20264) & $4145 \pm 30$ & $2876-2588$ \\
\hline
\end{tabular}

Tableau 1 : Dates radiocarbone AMS obtenues pour les sondages 2 et 3 (programme Artemis).

Table 1: AMS radiocarbon date obtained for the excavations 2 and 3 (Artemis program).

Calibration selon Reimer et al., 2009. Calibration from Reimer et al., 2009.

essences par N. Marcoux et D. Marguerie s'est faite dans le cadre de l'Action Collective de Recherche sur Locmariaquer (Table des Marchands, Grand Menhir).

Un charbon de vieux chêne $(15 \mathrm{~mm})$ provenant de la fosse de N15 devait contribuer à dater son comblement inférieur. Le résultat (4145 $\pm 30 \mathrm{BP}$; [2876-2588] av. J.-C. à $95 \%$ ) (tableau 1) s’inscrit dans le Néolithique final, mais nous semble moins fiable que le résultat suivant.

Un fragment de brindille bien conservée de noisetier (3 mm de diamètre) a été prélevé à la base de N6A, privilégié cette fois à d'autres charbons trouvés dans le comblement, pour sa durée de vie limitée. Le résultat nous porte dans le Bronze ancien $(3405 \pm 30 \mathrm{BP}$; [1752-1628] av. J.-C. à $95 \%)$ (tableau 1).

\section{Mise en œuvre de l'OSL}

L'événement que l'on cherche à dater est l'installation des blocs, c'est-à-dire la dernière exposition à la lumière des sédiments (lors de leur brassage) ou de la base des stèles (au moment de leur implantation dans la fosse). L'idée d'une datation de surface par Luminescence Stimulée Optiquement ou OSL (la pénétration de la lumière étant faible - quelques millimètres dans ces matériaux - on parle de datation de "surface ") est une idée récurrente dans la communauté. Depuis une vingtaine d'années, quelques tentatives ont été réalisées (Liritzis \& Vafiadou, 2005; Vieillevigne, 2006; Liritzis et al., 2007; 2010 ; Liritzis, 2011). Il s'agit d'une technique exploratoire qui nécessite d'importants développements méthodologiques (Tribolo et al., 2003) et en pratique, elle doit être adaptée à chaque situation, voire à chaque échantillon. Nous tentons ici d'évaluer les potentialités de son utilisation en vue de la datation directe de l'événement lié à l'érection des mégalithes.

\section{Choix des échantillons pour la datation par Luminescence Stimulée Optiquement}

Des prélèvements ont été réalisés dans le secteur occidental de l'alignement dans les sondages $\mathrm{n}^{\circ} 2$ et $\mathrm{n}^{\circ} 3$ correspondant respectivement aux menhirs N15 et N6A (fig. 6; tableau 2). Aucun des deux menhirs ne présentait de concrétions ni d'altérations à la fois dans leur partie visible et exposée et dans les parties enfouies qui ont été dégagées pour les sondages. La présence d'une altération de la roche, comme cela peut être le cas avec les granites, aurait en effet empêché la mise en œuvre de la datation de surface, c'est la raison pour laquelle ces deux éléments de l'alignement ont été choisis comme candidats à cette étude. Précisons que parmi les faciès variés de la roche décrite au paragraphe 2 , le menhir N6A est composé d'un quartz laiteux, particulièrement pur (99,5\% de SiO2), et le menhir N15 présente des passées sombres schisteuses. On observe quelques mousses et lichens en surface. Les deux roches sont donc du point de vue de leur transparence assez différentes et nous nous attendons à une plus grande transparence de N6A. Les prélèvements à la base des menhirs ont été effectués de nuit, pour éviter leur exposition à la lumière du jour. Les carottages des menhirs ont été réalisés sous lubrification à l'eau à l'aide d'un foret de $25 \mathrm{~mm}$ de diamètre interne.

Au niveau du menhir N15, des sédiments (référencés $\mathrm{Bdx} 12711$ et $\mathrm{Bdx}$ 12712) associés à la fosse d'implantation ont été échantillonnés à l'aide de tubes opaques. N15 a fait l'objet de plusieurs prélèvements par carottage. Un premier prélèvement a été effectué dans la partie exposée afin de vérifier la remise à zéro du signal d'OSL et de tester la profondeur de pénétration de la lumière. Dans les parties enfouies trois prélèvements ont été réalisés. Deux des carottes ont une longueur de $15 \mathrm{~mm}$, une troisième s'est fragmentée à la découpe sur le terrain. A posteriori, l'examen visuel des surfaces prélevées dans la base du menhir ne montrait aucune altération, ce qui garantissait la validité de l'échantillonnage.

Pour N6A, une carotte (Bdx 12708) a été prélevée de la partie émergente du bloc, sur une surface non altérée par d'éventuels organismes végétaux et non érodée. Cette zone a donc été exposée continument à la lumière, le but étant ici également de vérifier la remise à zéro correcte de la luminescence et d'estimer la profondeur de pénétration de la lumière dans le bloc comme il est d'usage pour ce type 

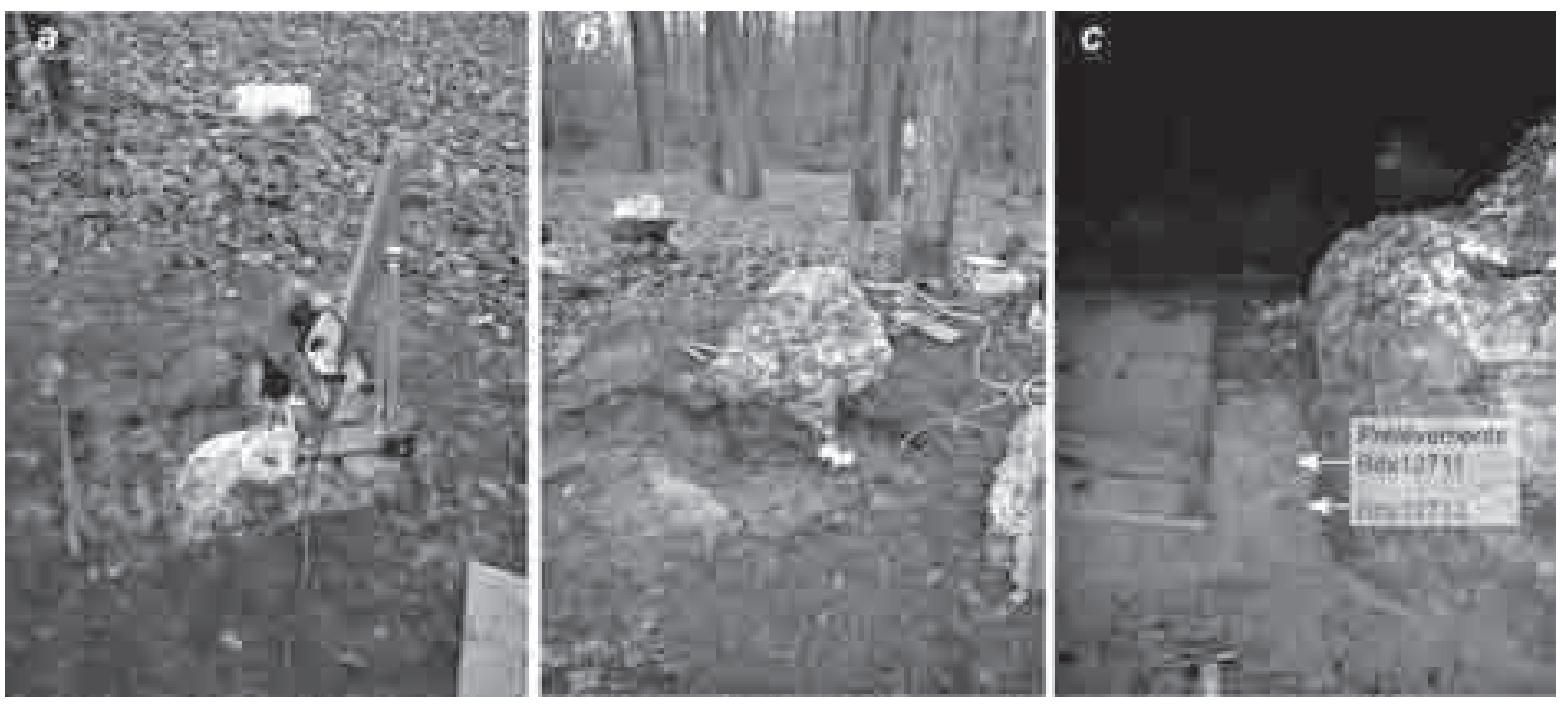

Fig. 6 (a, b et c) : (Voir planche couleur) Mise en situation du carottage de la pierre N6A dans la partie exposée; mise en place de la sonde gammamétrique à proximité de la roche N15; prélèvements de nuit des sédiments et de la pierre N15. Fig. 6 ( $a, b$ et c): (See colour plate) Overview of the core drilling of the stone N6A in the exposed part; implementation of the gammametric sensor near the rock N15; samples by night of sediments and stone N15.

\begin{tabular}{|c|c|c|c|}
\hline Sondage archéologique & $\mathrm{N}^{\circ}$ bloc & Description du prélèvement & Référence $\mathrm{Bdx}$ \\
\hline \multirow{4}{*}{$\mathrm{N}^{\circ} 2$} & \multirow{4}{*}{ N15 } & Surface supérieure du bloc, partie exposée à la lumière & Bdx 12709 \\
\hline & & $\begin{array}{l}\text { Surface inférieure du bloc, partie enfouie dans le sédiment } \\
\text { de la fosse, } 3 \text { carottes. Pas d'altération visible. }\end{array}$ & $\begin{array}{l}\text { Bdx } 12713 \\
\text { Bdx } 12714 \\
\text { Bdx } 12715\end{array}$ \\
\hline & & \multirow{2}{*}{$\begin{array}{l}\text { Deux échantillons de sédiment prélevés dans la fosse } \\
\text { d'implantation }\end{array}$} & Bdx 12711 \\
\hline & & & Bdx 12712 \\
\hline \multirow{3}{*}{$\mathrm{N}^{\circ} 3$} & \multirow{3}{*}{ N6A } & Surface supérieure du bloc, exposée à la lumière & Bdx 12708 \\
\hline & & $\begin{array}{l}\text { Surface inférieure du bloc, partie enfouie dans le sédiment } \\
\text { de la fosse, pas d'altération ni d'encroûtement ferrugineux }\end{array}$ & Bdx 12716 \\
\hline & & $\begin{array}{l}\text { Pierre de calage, découverte lors du creusement du sédi- } \\
\text { ment pour le carottage de la partie inférieure de N6A, de } \\
\text { même faciès que le bloc, pas d'altération }\end{array}$ & Bdx 12718 \\
\hline
\end{tabular}

Tableau 2: Tableau récapitulatif des prélèvements pour la datation.

Table 2: Summary table of the samples for the dating. d'étude (Vieillevigne et al., 2006; Laskaris et Liritzis, 2011). Un autre échantillon (Bdx 12716), également prélevé par carottage, provient de la partie inférieure et donc enfouie du menhir; on vise à évaluer la dose absorbée par la surface du bloc depuis sa dernière exposition à la lumière, à savoir depuis son enfouissement. Une pierre interprétée comme un élément de calage du menhir, a été récupérée dans le sédiment de la fosse d'implantation. L'objectif pour cette pierre est ici le même que pour l'échantillon précédent. Le contrôle a posteriori de la qualité de la surface échantillonnée a montré comme pour N15A l'absence d'altérations ou de concrétionnement sur les zones prélevées.

\section{Principe de la datation par OSL et mise en ouvre au laboratoire}

\section{Principe}

La méthode de datation par OSL est basée sur les phénomènes de luminescence et de radioactivité. En effet, les radioéléments contenus dans l'environnement émettent, lors de leur désintégration, des particules $\alpha, \beta$ et des rayonnements $\gamma$, lesquels, en conjonction avec les rayonnements cosmiques, irradient les cristaux de quartz contenus dans les menhirs ou les sédiments. Les quartz ont la propriété de stocker les effets de cette irradiation sous forme d'électrons 
piégés dans les défauts du cristal, et de libérer cette énergie cumulée sous forme de photons (c'est la luminescence) lors d'un apport d'énergie suffisant, tel qu'un éclairement (on parle alors de Luminescence Stimulée Optiquement ou OSL). De plus, la dernière exposition des grains à la lumière (avant leur dépôt ou enfouissement) permet la remise à zéro du chronomètre, puisqu' elle vide les pièges des cristaux. Une fois cachés de la lumière, ces cristaux absorbent de nouveau les effets de la radioactivité. Cette dernière étant considérée continue et constante au cours du temps, la dose totale absorbée par les grains de quartz, et donc l'intensité de la luminescence émise, sont proportionnelles à la durée d'exposition de l'échantillon. Ainsi on peut considérer l'équation d'âge suivante: $T=P / I$, où $T$ est le temps écoulé depuis la dernière exposition à la lumière jusqu'à nos jours (années), $\mathrm{P}$ est la dose archéologique totale absorbée par les grains de quartz ou exprimée en Gy ( $1 \mathrm{~Gy}=1 \mathrm{~J} / \mathrm{kg})$, et I, la dose fournie en un an par l'environnement de ces grains exprimée en Gy/an. La méthode consiste donc à déterminer ces deux paramètres physiques.

\section{Détermination de la dose annuelle}

Les différentes contributions de la dose annuelle dérivent du matériau lui-même (le bloc de pierre ou les sédiments encaissants) et de son environnement immédiat (le reste du sédiment, la stèle, les rayonnements cosmiques, etc.). Celle relative au matériau à dater est déterminée à partir des mesures de concentrations en potassium $(\mathrm{K})$, uranium $(\mathrm{U})$ et thorium (Th) des sédiments par spectrométrie gamma à bas bruit de fond avec un détecteur de type EGPC 200 P 17 (Eurisys Mesures) (Guibert et al., 2009). Les contributions gamma et cosmiques de l'environnement de l'échantillon sont évaluées par spectrométrie gamma de terrain à l'aide d'un spectromètre portable Canberra Inspector NaI.

\section{Détermination de la dose archéologique}

Les échantillons sont soumis à différents traitements mécaniques (broyage, tamisage) et chimiques (pour les attaques acides: $\mathrm{HCl} 1 \mathrm{M}$ pour les quartz des stèles; et pour les sédiments, $\mathrm{HCl} 1 \mathrm{M}$ suivi d'une attaque de longue durée avec un mélange $\mathrm{H}_{2} \mathrm{SiF}_{6}$ et $\mathrm{HNO}_{3}$ et d'un rinçage par $\mathrm{HCl}$ $1 \mathrm{M})$ pour ne récupérer qu'une seule phase cristalline: les quartz, de granulométrie donnée $(20-40 \mu \mathrm{m})$ pour les grains de quartz extraits des stèles et comprise entre 40 et $60 \mu \mathrm{m}$ pour les quartz des sédiments (granulométrie la plus représentée dans le sédiment analysé). Les grains de quartz ainsi sélectionnés sont ensuite déposés sur des disques en acier de $10 \mathrm{~mm}$ de diamètre et les mesures de luminescence sont réalisées sur ces disques à l'aide d'un lecteur d'OSL de marque DayBreak 2200, comprenant un tube photo- multiplicateur de type EMI 9635B, un filtre Hoya U340 $(7,5 \mathrm{~mm})$, et une source de stimulation constituée d'une diode bleue $\left(470 \mathrm{~nm} ;-50 \mathrm{~mW} \cdot \mathrm{cm}^{-2}\right)$. Le signal CW (Continued Wave) -OSL obtenu en réponse à une stimulation optique à puissance constante se présente sous la forme d'une courbe décroissante en fonction de la durée d'illumination (fig. 7) (voir aussi par exemple Bailey et al., 1997).

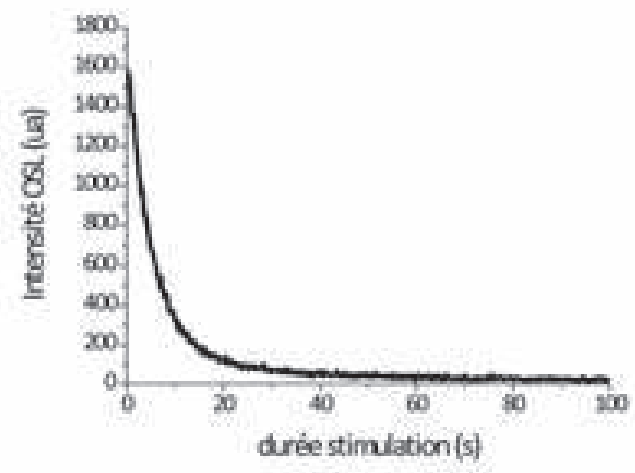

Fig. 7 : Signal de décroissance de l'OSL en fonction du temps. Fig. 7: Decay of the OSL signal as a function of time.

Le protocole utilisé pour déterminer la valeur de dose archéologique est un protocole standard dans la communauté appelé SAR (Single Aliquot Regeneration). Il permet de comparer sur une même prise d'essai (constituée de grains de quartz disposés sur un disque, aussi appelé aliquote), l'OSL naturelle résultant de l'irradiation naturelle de l'échantillon depuis sa dernière exposition à la lumière, et celles obtenues après régénération du signal par des doses d'irradiation connues (tableau 3). Ces dernières sont choisies de manière à encadrer la dose naturelle et sont administrées au laboratoire à l'aide d'une source bêta calibrée ${ }^{90} \mathrm{Sr} /{ }^{90} \mathrm{Y}$ délivrant une dose de 0,094 \pm 0,002 Gy/s au moment des expériences.

\section{Résultats de l'étude}

\section{Datation par OSL des sédiments}

La valeur moyenne de la dose archéologique déterminée par SAR et l'incertitude associée sont présentées dans le tableau 4 ainsi que les valeurs des concentrations en $\mathrm{K}$, $\mathrm{U}$ et Th mesurées en spectrométrie $\gamma$ à bas bruit de fond pour chaque échantillon et les différentes contributions à la dose annuelle. Les âges calculés (exprimés en années) sont également résumés dans ce tableau. La date de dernier éclairement est calculée par soustraction de l'année de la prise 


\begin{tabular}{|c|c|}
\hline Étape & Traitement \\
\hline 1 & Mesure de l'OSL naturelle (à $125^{\circ} \mathrm{C}$ pendant $\left.40 \mathrm{~s}\right)=\mathrm{L}_{\mathrm{n}}$ \\
\hline 2 & Irradiation par une dose-test $\mathrm{D}_{0}$ et mesure de l'OSL $=\mathrm{T}_{\mathrm{n}}$ \\
\hline 3 & $\begin{array}{c}\text { Irradiation par des doses dites de régénération }\left(\mathrm{D}_{\mathrm{x}}=\{1 ; 5 ; 9 ;\right. \\
14 ; 0 ; 1\} \text { Gy) }\end{array}$ \\
\hline 4 & Mesure de l'OSL (à $125^{\circ} \mathrm{C}$ pendant $\left.40 \mathrm{~s}\right)=\mathrm{L}_{\mathrm{x}}$ \\
\hline 5 & Irradiation par une dose-test $\mathrm{D}_{0}$ et mesure de l'OSL $=\mathrm{T}_{\mathrm{x}}$ \\
\hline 6 & Retour à l'étape 3 \\
\hline
\end{tabular}

Tableau 3: Séquence type d'un cycle de protocole SAR, appliqué aux grains des échantillons de menhir.

Table 3: Sequence-type of a cycle of SAR protocol, applied to the grains of the menhir samples.

des mesures au laboratoire (2010). Une valeur standard de $20 \pm 5 \%$ a été choisie pour l'humidité (avec des pourcentages massiques mesurés de l'humidité telle qu'au moment du prélèvement de $22,6 \%$ et $17,8 \%$ pour $\mathrm{Bdx} 12711$ et Bdx 12712 respectivement).

\section{Étude de l'OSL de surface des menhirs}

À la différence des quartz extraits des sédiments étudiés, ceux des menhirs émettent un signal nettement moins intense et donc beaucoup moins reproductible, comme le montre la courbe d'OSL d'une aliquote naturelle d'un échantillon de sédiment comparée à celle d'un échantillon du menhir N6A (fig. 8).

Sensibilité OSL des roches et aptitude à la datation

Les propriétés intrinsèques des grains de quartz du menhir N6A ont été examinées. Pour ce faire, nous avons utilisé une méthode de stimulation non plus continue mais augmentée linéairement; il s'agit de LM (Linearly Modulated) -OSL. Cette méthode permet de différencier les différentes contributions du signal d'OSL (Smith \& Rhodes, 1994) en entraînant la libération progressive des charges par ordre décroissant de section efficace d'interaction entre les photons et les charges piégées (fig. 9).

Le contraste notable d'intensité des courbes de LM-OSL confirme l'extrême faiblesse de la sensibilité de l'OSL des grains de quartz des menhirs observée en CW-OSL. On constate aussi que les composantes prépondérantes de l'OSL des quartz des menhirs, sont les composantes dites rapides de l'OSL, généralement aptes à la datation (si elles étaient plus intenses) en raison de leur stabilité thermique suffisante. Les échantillons du menhir N6A ne sont toutefois pas totalement insensibles à l'irradiation puisque l'OSL révèle une réponse faible mais non nulle à la dose lorsque l'on combine les signaux issus des différentes aliquotes de l'échantillon (fig. 10).

\begin{tabular}{|c|c|c|c|c|c|c|c|c|c|c|c|c|}
\hline Éch. & $\begin{array}{l}\mathrm{P} \pm \sigma \\
(\mathrm{Gy})\end{array}$ & $\begin{array}{l}\mathrm{U}\left({ }^{(238} \mathrm{U}\right) \\
(\mathrm{ppm})\end{array}$ & $\begin{array}{c}\mathrm{U}\left({ }^{226} \mathrm{Ra}\right) \\
(\mathrm{ppm})\end{array}$ & $\begin{array}{c}\mathrm{U}\left({ }^{(210} \mathrm{Pb}\right) \\
(\mathrm{ppm})\end{array}$ & K (\%) & $\begin{array}{c}\text { Th } \\
\text { (ppm) }\end{array}$ & $\mathrm{I}_{\alpha} \pm \sigma$ & $\mathrm{I}_{\beta} \pm \sigma$ & $\mathrm{I}_{\mathrm{env}} \pm \sigma$ & $\mathrm{I}_{\text {tot }} \pm \sigma$ & Âge (ans) & $\begin{array}{l}\text { Date } \\
(\mathrm{BC}) \pm \sigma \\
\text { (stat) }\end{array}$ \\
\hline $\begin{array}{c}\text { N15 } \\
\text { Bdx } 12711\end{array}$ & $\begin{array}{c}4,89 \pm \\
0,07\end{array}$ & $\begin{array}{c}2,43 \pm \\
0,15\end{array}$ & $\begin{array}{c}2,29 \pm \\
0,03\end{array}$ & $\begin{array}{c}2,45 \pm \\
0,22\end{array}$ & $\begin{array}{c}0,91 \pm \\
0,02\end{array}$ & $\begin{array}{c}8,69 \pm \\
0,11\end{array}$ & $\begin{array}{c}0,14 \pm \\
0,02\end{array}$ & $\begin{array}{c}1,03 \pm \\
0,06\end{array}$ & $\begin{array}{c}1,09 \pm \\
0,02\end{array}$ & $\begin{array}{c}2,26 \pm \\
0,11\end{array}$ & $\begin{array}{c}2163 \pm \\
102\end{array}$ & $\begin{array}{c}153 \pm \\
102(34)\end{array}$ \\
\hline $\begin{array}{c}\text { N15 } \\
\text { Bdx } 12712\end{array}$ & $\begin{array}{c}9,13 \pm \\
0,11\end{array}$ & $\begin{array}{c}2,51 \pm \\
0,41\end{array}$ & $\begin{array}{c}2,62 \pm \\
0,03\end{array}$ & $\begin{array}{c}2,60 \pm \\
0,23\end{array}$ & $\begin{array}{c}0,98 \pm \\
0,02\end{array}$ & $\begin{array}{c}9,39 \pm \\
0,11\end{array}$ & $\begin{array}{c}0,15 \pm \\
0,02\end{array}$ & $\begin{array}{c}1,11 \pm \\
0,06\end{array}$ & $\begin{array}{c}1,09 \pm \\
0,02\end{array}$ & $\begin{array}{c}2,36 \pm \\
0,11\end{array}$ & $\begin{array}{c}3870 \pm \\
181\end{array}$ & $\begin{array}{c}1860 \pm \\
181(52)\end{array}$ \\
\hline
\end{tabular}

Tableau 4: Données expérimentales et détermination de l'âge OSL.

Table 4: Experimental data and OSL age determiantion.

Valeur de la dose archéologique. Composition radiochimique des sédiments du Gâvre, où $U\left({ }^{238} U\right)$ représente la teneur en uranium " tête de série de l'uranium » déduite des émissions de ${ }^{234} \mathrm{Th},{ }^{234 \mathrm{~m}} \mathrm{~Pa}$ et ${ }^{235} \mathrm{U}$, et $\mathrm{U}\left({ }^{226} \mathrm{Ra}\right)$ correspond aux teneurs déterminées à partir des descendants directs de ${ }^{226} \mathrm{Ra},{ }^{214} \mathrm{~Pb}$ et ${ }^{214} \mathrm{Bi}$, en supposant l'équilibre entre ${ }^{222} \mathrm{Rn}$ et ${ }^{226} \mathrm{Ra}$. Calcul de la dose annuelle totale ( $\left.\mathrm{I}_{\text {totale }}\right)$ exprimée en $\mathrm{mGy} / \mathrm{an}$ à partir des contributions internes $\left(\mathrm{I}_{\alpha}\right.$ et $\left.\mathrm{I}_{\beta}\right)$ et environnementale $\left(\mathrm{I}_{\text {env }}\right)$. Résultats de la datation par OSL des sédiments du Gâvre. Les incertitudes correspondent à un écart-type total intégrant quadratiquement l'incertitude statistique propre aux mesures individuelles et l'incertitude systématique qui concerne essentiellement les erreurs d'étalonnage et d'évaluation de l'humidité moyenne du sol depuis la mise en place des menhirs.

Value of the archaeological dose. Radiochemical composition of sediments of Gâvre, where $U\left({ }^{38} U\right)$ represents the content in uranium "head of $U$ series" deduced from the emissions of ${ }^{234} \mathrm{Th},{ }^{234 \mathrm{~m}} \mathrm{~Pa}$ and ${ }^{235} \mathrm{U}$, and $U\left({ }^{26} \mathrm{Ra}\right)$ corresponds to the contents determined from the direct descendants of ${ }^{226} \mathrm{Ra},{ }^{214} \mathrm{~Pb}$ and ${ }^{214} \mathrm{Bi}$, by supposing the equilibrium between ${ }^{222} \mathrm{Rn}$ and ${ }^{226} \mathrm{Ra}$. Calculation of the total annual dose (Iotale) expressed in $\mathrm{m} G y / y e a r$ from the addition of internal contributions $\left(I_{\alpha}\right.$ and $\left.I_{\beta}\right)$ and environmental $\left(I_{\text {en }}\right)$. OSL dating results of sediments of le Gavre. The uncertainties correspond to one total standard deviation calculated by the quadratic sum of the statistical standard deviation which is related to the individual measures; and the systematic uncertainty which regroups essentially the calibration errors and the estimated one in the evaluation of the average bumidity of the ground since the erection of menhirs. 


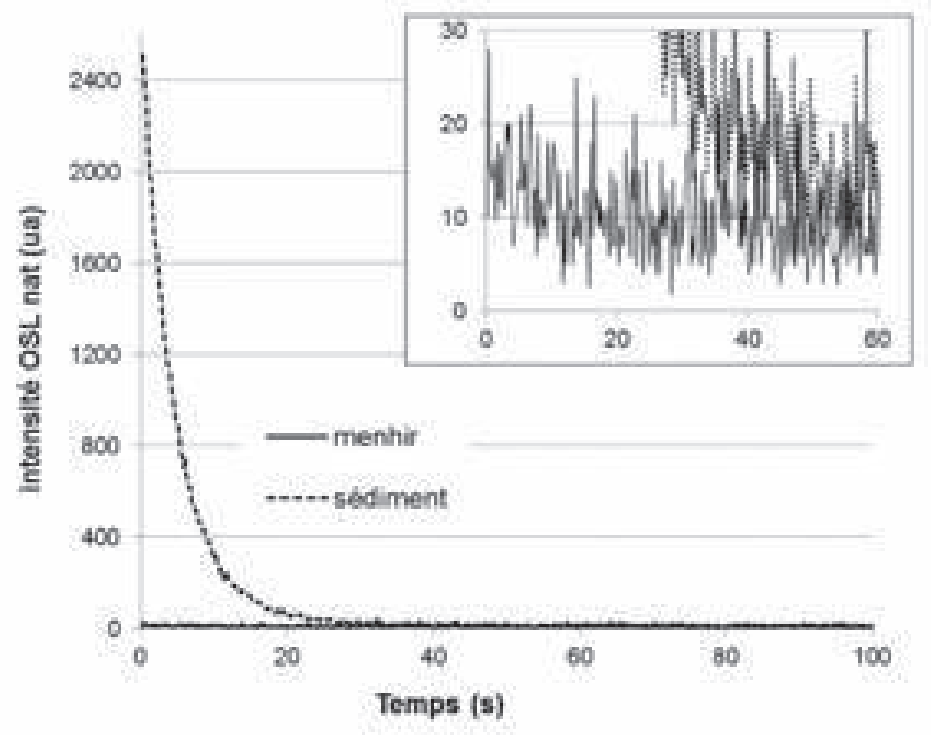

Fig. 8: Signal d'OSL naturel d'une aliquote de menhir (Bdx 12716) et de celui d'un sédiment ( $\mathrm{Bdx}$ 12711); en encadré: zoom sur le début du signal d'OSL du menhir.

Fig. 8: Signal of natural OSL of an aliquot of menhir (Bdx 12716) and that of the sediment (Bdx 12711); in box: zoom over the beginning of the signal of OSL of the menhir.
L'échantillon Bdx 12709 issu du menhir N15, quant à lui, émet un signal d'OSL trop faible pour être exploité, même en regroupant les données issues de plusieurs disques. Aussi, nous avons décidé de ne poursuivre l'étude que sur la roche N6A (quartz laiteux).

Répartition de la dose archéologique apparente avec la profondeur du prélèvement par rapport à la surface des roches

Ce comportement partiellement exploitable de l'OSL de N6A nous autorise donc à tenter de caractériser de l'évolution du signal d'OSL en fonction de la profondeur dans le bloc et dans la pierre de calage. Le tableau 5 regroupe les données obtenues en considérant pour chaque échantillon trois zones distinctes selon leur position par rapport à la surface.

On constate que pour l'échantillon exposé en continu à la lumière (Bdx 12708), la dose apparente en surface n'est pas rigoureusement nulle, mais elle reste néanmoins très significativement plus faible que pour les parties enfouies Bdx 12716 et 12718. Il convient de préciser que l'incertitude associée à la mesure de dose représente un seul écart-type et que celui-ci est calculé à partir de la dispersion des points expérimentaux par rapport à la courbe d'étalonnage de l'OSL. Ainsi, une bonne convergence de l'ensemble des données pour la surface de Bdx 12708 qui résulte d'un artefact statistique favorable, implique-t-elle une incertitude faible en apparence $(0,1 \mathrm{~Gy})$ alors que toutes les autres mesures ont un écart-type compris entre 0,3 et 0,6 Gy davantage compatible avec la proximité de l'intensité des signaux avec la limite de détection. Cette observation, malgré les fortes incertitudes affectées à la dose apparente résiduelle, tendrait à montrer que les grains de la carotte $\mathrm{Bdx} 12708$ n'auraient pas été complètement blanchis, conclusion évidemment à nuancer par rapport à la forte incertitude de mesure et qui devra être confirmée lors d'une prochaine étude de ce type.

Lexamen des doses obtenues selon la partie de la carotte étudiée reste néanmoins possible. Tout d'abord, toutes les doses sont largement inférieures aux doses de saturation (150 Gy voire davantage) auxquelles nous sommes habitués pour des quartz non ou faiblement blanchis. Nous devons donc considérer que la lumière a eu des effets sensibles sur la totalité des deux prélèvements du menhir (Bdx 12708 et 12716) et de la pierre de calage ( $\mathrm{Bdx}$ 12718). La transparence de ces roches, les conditions de leur exposition ancienne et la dimension limitée des carottes ne nous ont pas permis d'atteindre des zones internes non affectées par la lumière, mais ce que nous cherchions avant tout était l'existence d'une zone blanchie de dimension suffisante pour atteindre des zones hors de portée de particules béta issues du sol (quelques millimètres).

Pour Bdx 12716, les doses tendent à décroître de la surface vers l'intérieur (jusqu'à $8 \mathrm{~mm}$; dimension de la carotte). Il n'a pas été possible de disposer d'un échantillon plus grand en raison de la dureté de la roche et des conditions de prélèvement, aussi nous devons considérer que l'effet de l'exposition ancienne de surface a donc été supérieur à $8 \mathrm{~mm}$. La décroissance de la dose archéologique entre la partie superficielle du prélèvement (moins de $2,5 \mathrm{~mm}$ ) et la partie interne (entre 2,5 et $8 \mathrm{~mm}$ ) est compatible avec l'atténuation des 


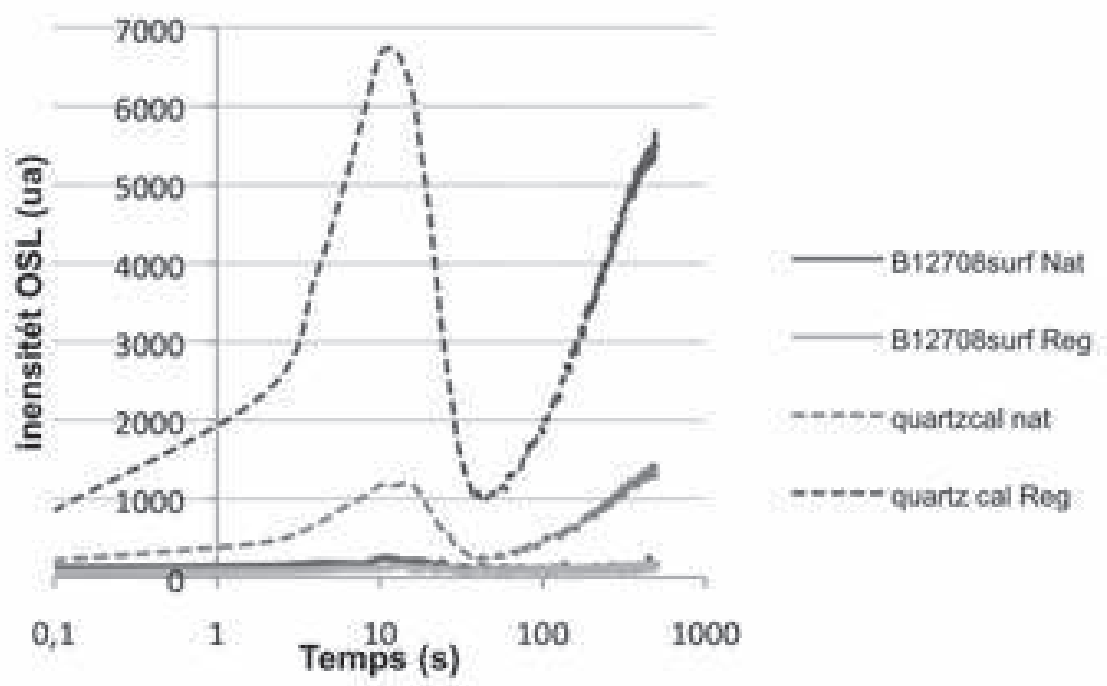

La courbe présente plusieurs pics qui apparaissent par ordre de blanchiment décroissant des différentes composantes du signal d'OSL: le premier sommet correspond à la somme des composantes dites "rapides et moyennes ", et le second, à la somme des composantes "lentes ». On observe une grande différence d'intensité et d'homothétie des courbes de LM-OSL des quartz du menhir par rapport à un quartz de référence (quartz de calibration distribué par le constructeur d'équipements d'OSL Risoe).

The curve presents several peaks which appear in order of decreasing bleaching of the various components of the signal of OSL: the first peak corresponds to the sum of the so-called "fast and average" components, and the second, to the sum of the "slow" components. We observe a clear difference of intensity and homothety of the curves of LM-OSL of the quartz from the menhir compared with a quartz reference (calibration quartz distributed by the OSL reader manufacturer Risoe).
Fig. 9: Comparaison des signaux de LM-OSL (signaux naturel et régénéré après une irradiation de 4 Gy) entre un quartz de calibration (tracé en pointillé) et des grains de quartz du menhir N6A de l'échantillon Bdx 12708 (tracé en continu).

Fig. 9: Comparison of the signals of LM-OSL (natural and regenerated signals after an irradiation of 4 Gy) between a quartz of calibration (drawn in dotted line) and grains of quartz of the menhir N6A from the Bdx 12708 sample (drawn continuously).

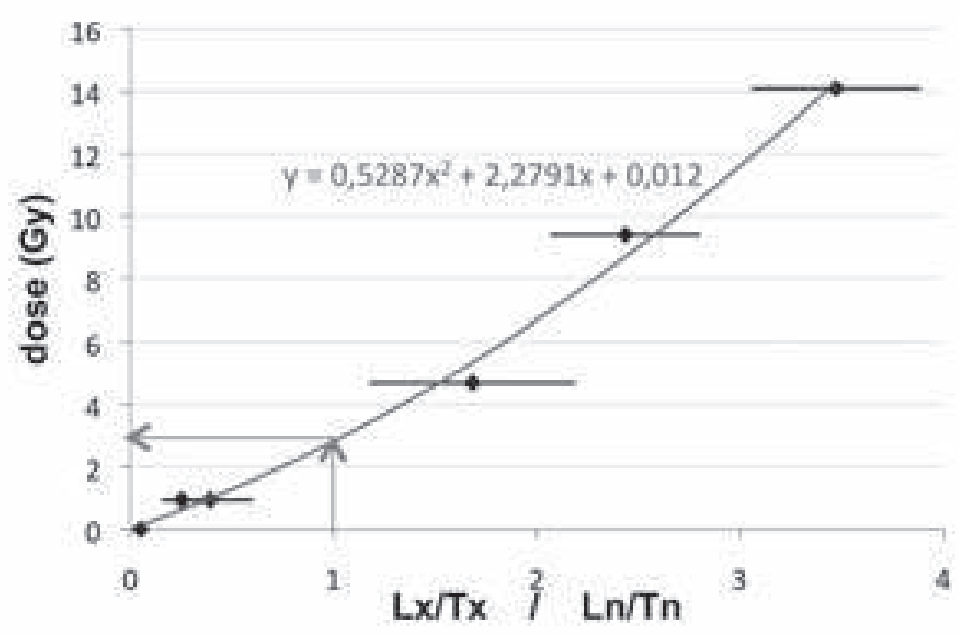

Fig. 10: Loi de croissance de la dose ajoutée en fonction du signal d'OSL normalisé pour l'échantillon Bdx 12708, en surface.

Fig. 10: Growth curve of OSL with dose for sample $B d x 12708$, sample form the surface. OSL signals are normalised to the natural one.

Compte tenu des incertitudes, la dose équivalente que l'on peut déduire de cette courbe (obtenue pour la valeur 1 de l'abscisse) sera comprise entre 2 et 4 Gy. Lx et Ln sont respectivement les intensités d'OSL du signal régénéré par une dose $\mathrm{x}$ et le signal naturel. Tx et Tn sont respectivement les intensités d'OSL obtenues par des irradiations avec des doses tests constantes consécutives aux mesures de Lx et Ln.

Considering the uncertainties, the equivalent dose amounts we can deduce from this curve (obtained for the value 1 of the abscissa) will be included between 2 and $4 G y . L x$ and $L n$ are respectively the intensities of OSL of the signal regenerated by a dose $x$ and the natural signal. $T x$ and $T n$ are respectively the intensities of OSL obtained after irradiation by constant test doses given just after the measures of $L x$ and $L n$. 


\begin{tabular}{|c|c|c|c|c|c|c|}
\hline & \multicolumn{2}{|c|}{ Bdx 12708 (partie exposée) } & \multicolumn{2}{|c|}{ Bdx 12716 (partie enfouie) } & \multicolumn{2}{|c|}{ Bdx 12718 (pierre de calage) } \\
\hline & Prof. (mm) & $\mathrm{P}_{\text {app }}(\mathrm{Gy})$ & Prof. (mm) & $P_{\text {app }}(G y)$ & Prof. (mm) & $\mathrm{P}_{\text {app }}(\mathrm{Gy})$ \\
\hline Surface & de 0 à $0,5 \mathrm{~mm}$ & $1,1 \pm 0,1(?)$ & de 0 à $2,5 \mathrm{~mm}$ & $3,3 \pm 0,4$ & de 0 à $3,5 \mathrm{~mm}$ & $3,2 \pm 0,3$ \\
\hline Interface & de 0,5 à $4 \mathrm{~mm}$ & $0,8 \pm 0,3$ & de 2,5 à $5,5 \mathrm{~mm}$ & $2,5 \pm 0,6$ & de 3,5 à $6,5 \mathrm{~mm}$ & $2,9 \pm 0,4$ \\
\hline Intérieur du bloc & de 4 à 9 mm & non-mesurable & de 5,5 à $8 \mathrm{~mm}$ & $2,1 \pm 0,3$ & de 6,5 à $10,5 \mathrm{~mm}$ & $4,8 \pm 0,6$ \\
\hline
\end{tabular}

Tableau 5.Valeur de la dose archéologique apparente ( $\mathrm{P}_{\text {app }}$ ”, en Gy) calculée pour les trois échantillons relatifs au menhir N6A, en fonction de la profondeur ("Prof.»), de la surface vers l'intérieur du bloc.

Table 5. Value of the apparent archaeological dose ("Papp ", in Gy) calculated for three samples relative to the menhir N6A, according to the depth ("Prof."), from the surface inward the internal part of the block.

béta issus du sédiment et le fait que le quartz ne soit pratiquement pas radioactif.

Pour la pierre de calage (Bdx 12718), constituée du même matériau que le menhir N6A, des remarques similaires peuvent être faites: on observe en effet une décroissance de la dose entre la partie superficielle (moins de $3,5 \mathrm{~mm}$ ) et une partie plus interne (entre 3,5 et 6,5 $\mathrm{mm}$ ). L'augmentation sensible de la dose archéologique mesurée pour la partie encore plus interne, peut être interprétée par l'accès à des zones de la roche moins exposées dans le passé et présentant donc une remise à zéro partielle. Enfin, on notera la convergence des doses de surface de ces deux échantillons (3,2-3,3 Gy) et des doses des parties intermédiaires (2,52,9 Gy), ce qui dénote de la reproductibilité satisfaisante du comportement de ces matériaux, même avec la faible sensibilité qui les caractérise.

\section{VERS UNE CHRONOLOGIE DE L'ALIGNEMENT MÉGALITHIQUE DU GÂVRE}

La datation de sédiments par OSL effectuée dans cette étude est une des premières tentatives en France de datation indirecte de mégalithes à partir des sédiments. Au vu des résultats obtenus, les deux prélèvements datés sont en bonne corrélation avec la séquence stratigraphique (C3 et C4); la couche supérieure du comblement de la fosse est contemporaine de l'âge du Fer, ou polluée par des mélanges avec les dépôts tardifs. En effet, une dizaine de centimètres sépare les deux prélèvements, Bdx 12712 étant le plus profond (fig. 2). Ainsi, l'intervalle de dates [2220-1500] av. J.-C. (à $2 \sigma$ ) obtenu pour l'échantillon Bdx 12712 situe le creusement de la fosse et donc l'érection du bloc au Bronze ancien. Cette datation est en accord avec celle obtenue indirectement pour le bloc N6A $(3405 \pm 30$ BP soit [17501630 ] av. J.-C. à 2) grâce à l'analyse ${ }^{14} \mathrm{C}$ d'un charbon de bois. Cette bonne concordance tend à valider les deux dates. Quant à l'intervalle de dates [360 av. J.-C. - 50 apr. J.-C.] (à $2 \sigma$ ) obtenu pour l'échantillon Bdx 12711, celui-ci suggère plutôt un remaniement survenu à l'âge du Fer (la Tène), une occupation reconnue dans la forêt du Gâvre (Meuret 2001; Carte archéologique, DRAC Pays de Loire).

Cependant, il est important de préciser que ces interprétations se basent sur l'hypothèse selon laquelle l'ensemble du sédiment a bien vu le jour; c'est-à-dire que l'ensemble des grains a été mis au jour au moment du rebouchage de la fosse. Pour valider définitivement cette hypothèse, il s'avérerait nécessaire d'avoir recours à la technique du « monograin » qui permet de mesurer la luminescence émise individuellement par chaque grain. Cependant la granulométrie majoritaire du sédiment du Gâvre ne se prête pas à ce type d'analyse qui nécessite des grains d'environ $200 \mu \mathrm{m}$ de dimensions caractéristiques.

\section{ConClusions ET DISCUSSION}

Le vaste ouvrage de pierres dressées dit du Pilier en forêt du Gâvre, un des plus longs d'Europe (une centaine de blocs préservée sur au moins $900 \mathrm{~m}$ ), nécessitait d'être requalifié après avoir été déclaré d'origine naturelle (Carte archéologique, SRA Pays de Loire, diagnostic 1976, J. L'Helgouac'h) alors que paradoxalement la carte géologique le signalait comme mégalithe (1987, BRGM carte $\mathrm{n}^{\circ} 420$ ). Cette redéfinition imposait une étude géologique des blocs et du substrat local, et une proposition de date. Plus généralement, ces structures architecturales s'inscrivent dans un renouvellement de la problématique des barres de stèles dont un modèle théorique a été récemment proposé à partir des données morbihanaises, et notamment celles de l'alignement dit du Grand Menhir découvert en 1989 à Locmariaquer (Cassen, 2009a). 
Trois sondages restreints au contact de trois blocs (1 couché, 2 dressés) ont bien détecté des fosses d'implantation pour les deux monolithes plantés (N6A et N15). Les stratigraphies relevées ont également montré l'implantation des pierres dans un contexte sédimentaire d'altération au contact du substrat schisteux. Ce substrat, non atteint dans ces sondages, apparaît cependant à $40 \mathrm{~cm}$ de profondeur dans un autre sondage à $1 \mathrm{~m}$ au nord-ouest du bloc N34 B. La majorité des stèles plantées est en quartz blanc laiteux, stèles plus rarement composées de schistes gris-vert. L'ouvrage du Pilier est bel et bien d'origine anthropique.

Les files de blocs quartzitiques mettent donc à profit une singularité du sous-sol, un filon à l'époque probablement visible en surface à travers diverses émergences de quartz blanc, comme on peut aujourd'hui encore les découvrir en limite sud du massif forestier (secteur de Curun). L'orientation de l'ouvrage suit de surcroît la direction du filon naturel de quartz bien noté dans son prolongement, $1,5 \mathrm{~km}$ plus à l'ouest, à l'azimut $110^{\circ}$. On se gardera pourtant de conclure que la direction tectonique a impérativement dicté l'implantation et l'orientation de la structure architecturale. Un ouvrage à dimension pour le moins symbolique, à l'image d'une barre de stèles développée à une telle échelle, relève de motivations socio-religieuses complexes qui peuvent bien entendu intégrer et inclure les formes du relief et la nature sensible des matériaux. Ainsi, la barre du Grand Menhir coïncide exactement en direction avec une grande diaclase visible à cet endroit dans le granite de Locmariaquer; ou encore, l'« Avenue » de fossés et talus connectée au fameux Stonehenge est exactement parallèle aux sillons périglaciaires bien notés dans le substrat (Cassen, 2009b; Parker Pearson et al., à paraître). Mais les contre-exemples à propos de ces constructions sont encore plus abondants et tempèrent en définitive toute généralisation et tout déterminisme géologique ou pédologique. Si le phénomène tectonique observé au Gâvre a très nettement influencé l'emprunt direct des roches et l'orientation de leur implantation (après tout, tectonique vient du grec $\tau \dot{\varepsilon} \kappa \tau \omega v$ ou tektōn signifiant "bâtisseur "...), le site peu éloigné du Moulin (Saint-Just, à $15 \mathrm{~km}$ au NO) témoigne de l'importation de blocs de quartz filonien sur une distance de $3 \mathrm{~km}$, avant d'être dressés et intégrés à trois files d'orientations distinctes (Le Roux et al., 1989), nous rappelant ainsi que ces architectures dépendent toutes d'un modèle anthropologique pour les animer.

La chronologie de la construction est l'autre nécessité permettant de requalifier l'ouvrage. Devant l'absence de référentiel dans l'Ouest de la France pour dater ces structures particulières en roches quartzitiques, toutes éloignées de la concentration carnacoise bien calée maintenant dans le milieu et la seconde moitié du Ve millénaire (Cassen et al., à paraître), la méthode OSL semble une approche très intéressante pour rapidement lever l'incertitude historique de la fondation, voire même la période de destruction. La classique fouille est en effet contrainte par le gigantisme des ouvrages et le diagnostic mené sur du mobilier trouvé aux pieds des stèles, trop souvent isolé de son environnement, semble d'une pertinence discutable (cf. les pièces de monnaie romaines ou modernes enfouies à leurs bases - Cassen, 2009a).

Encadré par une date radiocarbone sur charbon présentant d'excellentes garanties, en provenance de la fosse voisine N6A [1750-1630] av. J.-C., la date OSL opérée sur les sédiments comblant la fosse du monolithe N15 renvoie elle aussi au Bronze ancien [2040-1680] av. J.-C. Cette date est, notons-le, conforme à l'occupation secondaire du tumulus peu éloigné du Château-Bû (Saint-Just) sur lequel des stèles en quartz blanc furent dressées au Bronze ancien (Briard et al., 1995). Les quartz des sédiments de l'ouvrage du Pilier émettent donc un signal suffisant pour être exploité à des fins de datation. Ce n'est malheureusement pas le cas pour les deux monolithes testés directement; le quartz en surface de ces deux stèles du Gâvre n'est pas adapté à la datation de surface par OSL en raison d'une trop faible sensibilité à l'irradiation liée, dans cette roche précisément, à la nature des systèmes de défauts et d'impuretés. Un test de sensibilité à l'irradiation s'impose donc sur tout monolithe avant de développer un protocole complet. On évitera également de prélever sur des menhirs en calcaire (le signal OSL n'étant pas évident, le risque de confusion avec de la photoluminescence de la calcite étant trop élevé), mais grès et granites offrent à l'évidence en Armorique un nouveau et vaste champ d'investigation.

Les tentatives menées ces dernières années en Europe, en contextes de mégalithes, sont globalement encourageantes (Grau Bitterli, Fierz-Dayer, 2011; López-Romero, 2011), bien que parfois surexploitées au regard des données disponibles. Au Portugal, une stèle en granite supposée néolithique, à Quinta da Queimada (Lagos), a fait l'objet de deux datations OSL à deux hauteurs distinctes dans le remplissage de la fosse de fondation (Calado et al., 2003). L'écart entre la première date obtenue près de la surface (Shfd 2013: $5925 \pm 175$ ) s'inscrivant entre le $V^{e}$ et le IV ${ }^{e}$ millénaire av. J.-C., et la seconde en profondeur (Shfd 2014: $9095 \pm 445$ av. J.-C.) est sans doute important, mais plus problématique est l'ancienneté de cette dernière qui ne peut être encore sérieusement corrélée à un groupe humain déterminé, malgré l'interprétation des fouilleurs (Calado et al., 2010). Un autre écart sépare deux dates OSL obtenues du remplissage de la fosse d'implantation de l'énorme dalle dressée en grès ("Cove Stone ») à Avebury 
en Angleterre (Gillings et al., 2008). Une première date marquant la transition $\mathrm{IV}^{e}-\mathrm{III}^{e}$ millénaire $( \pm 350)$ - très valorisée, elle aussi, par les commentateurs car antérieure à Stonehenge - accompagne l'autre date cette fois placée en plein âge du Fer (dont les témoignages sont en effet présents sur le site). Une situation qui, finalement, n'est pas sans rappeler le résultat de la forêt du Gâvre.

\section{Remerciements}

Nous aimerions remercier G. San Juan, CRA des Pays de Loire, qui a tenu à faire aboutir ce projet, après un rejet de la CIRA Grand Ouest. Les bénévoles de la Maison de la Forêt à Gâvre et les membres du CERAPAR (B. Monnier) ont fourni un travail considérable pour dégager une bande de végétation de $20 \mathrm{~m}$ de large sur près d'1 km de long, et nous ont efficacement aidé dans la réalisation du plan topographique. Les sondages ont été menés grâce à l'aide déterminante de C. Bidaud, E. Geslin, Y. Kernaleguen, J.-P. Leroux, V. Torgunakov; M. Mabilais a assuré un enregistrement vidéo de la campagne 2008. L'ONF a bien voulu tenir compte de nos recommandations dans la coupe et le débardage des secteurs concernés par les mégalithes. D. Marguerie et N. Marcoux (CNRS, Rennes) ont très aimablement déterminé les deux charbons prélevés et envoyés à la mesure radiocarbone. C. Oberlin (UMR 5138 Archéométrie et Archéologie CNRS - universités Lyon 1 et 2) a spontanément proposé de donner une seconde chance à l'échantillon $\mathrm{N} 15$ et nous lui en sommes reconnaissants. D. Calado (IPPAR) a bien voulu nous guider en 2006 lors de la mise en place de ce projet. L'équipe bordelaise tient égalent à remercier Victoria Kostenska, étudiante en master des Matériaux du Patrimoine à l'université de Bordeaux 3 au moment de l'étude (en 2010), et Marion Hernandez, post-doctorante à I'IRAMAT-CRP2A. Un grand merci enfin à Agnès Baltzer et Jean-Marc Rousset pour leur accueil chaleureux.

Pour l'IRAMAT-CRP2A, les institutions suivantes ont participé au financement de cette opération (salaires, équipements, fonctionnement): CNRS, Université Bordeaux 3, PRES université de Bordeaux, Conseil régional d'Aquitaine.

\section{Bibliographie}

Bailey, R.M., Smith B.W., Rhodes E.J., 1997. Partial bleaching and the decay form characteristics of quartz OSL, Radiation Measurements 27: 123-136.

Boujot, C., Pinet, L., 2007. Mégalithes et pierres dressées, matériau du discours scientifique en Préhistoire: évolution et perspectives d'après les exemples de Carnac (Bretagne) et du plateau de Cauria (Corse). In J. Évin (dir.). Congrès du centenaire de la S.P.F., Un siècle de construction du discours scientifique en Préhistoire, Société préhistorique française, Vol. 3: 185-197.

Briard, J., Gautier, M., Leroux, G., 1995. Les mégalithes et les tumulus de Saint-Just, Ille-et-Vilaine: évolution et acculturations d'un ensemble funéraire, 5000 à 1500 ans avant notre ère. Éd. du Comité des travaux historiques et scientifiques Documents préhistoriques, 8 , CTHS, Ministère de l'éducation nationale, Paris.

Calado, D., Nieto Liñán, J. M. et Nocete Calvo, F., 2003. Quinta da Queimada, Lagos, Portugal. Datação do momento de erecção de um monumento megalítico através da luminescência óptica de cristais de quartzo (OSL), V Congreso Ibérico de Arqueometría. Libro de Resúmenes de Actas (Puerto de Santa María, Cádiz): 167-168.

Calado, D., Càmalich, M. D., Martín-Socas, D., Nieto, J. M., Delgado, A., Nocete, F., Rodríguez, A., Bayona, M., Alex, E., InÁcio, N., 2010. Some Stones Can Speak! The social structure, identity and territoriality of SW Atlantic Europe complex appropriator communities reflected in their standing stones. In D. Calado, M. Baldia, M. Boulanger (dir.). Monumental Questions: Prehistoric Megaliths, Mounds, and Enclosures. Proceedings of the XV World Congress UISPP (Lisbon, 4-9 September 2006) 7 Session C68 (Part I), BAR S2122: 7-15.

Cassen, S. 2009a. Exercice de stèle. Une archéologie des pierres dressées. Réflexions autour des menhirs de Carnac. Éd. Errance, Paris.

Cassen, S., 2009b. La simulation des faits imaginés: phases, séquences, scénarios historiques autour d'une barre de stèles et d'une tombe à couloir. In S. Cassen (dir.). Autour de la Table. Explorations archéologiques et discours savants sur des architectures néolithiques à Locmariaquer, Morbihan (Table des Marchands et Grand Menhir). Actes du colloque international, Vannes, université de Bretagne-Sud, 5-7 octobre 2007. Université de Nantes, LARA: 881-909.

CAssen, à paraître. Sites de passage (1). Le modèle carnacois des pierres dressées à l'épreuve des rivières, des lacs et des montagnes (France, Suisse, Italie). In R.M. Arbogast, A. Richard (dir.), Hommages à Pierre Pétrequin, CNRS, DRAC FrancheComté.

Cassen, S., Boujot, C., Baltzer, A., Bonniol, D., Chaigneau, C., Dardignac, C., François, P., Guibert, P., Hinguant, S., Lanos, P., Leroux, V.-E., Lorin, A., Marguerie, D., Menier, D., Robin, G., 2009. Recherches archéologiques en cours sur les ouvrages de pierres dressées en Armorique-sud. In Journée du "CReAAH "Archéologie, Archéosciences, Histoire. Université Rennes 1: 6-11.

Cassen, S., Boujot, C., Blanchet, S., Dardignac, C., Hinguant, S., Fromont, N., Large, J.M., Lorho, T., à 
paraître. Méthodes d'investigations des ouvrages de stèles dans l'ouest de la France (1987-2012). In I. SÉnÉPART, C. Billard, Y. Praud (dir.). Méthodologie des recherches sur la Préhistoire récente en France: nouveaux acquis, nouveaux outils (19872012), Marseille 23-24-25 mai 2012, RPM et Internéo.

Gillings, M., Pollard, J., Wheatley, D. W. et Peterson, R., 2008. Landscape of the Megaliths: excavation and fieldwork on the Avebury monuments, 1997-2003, Oxbow Books, Oxford.

Grau Bitterli, M.-H., Fierz-Dayer, E. (dir.), 2011. Plateau de Bevaix 6, Bevaix/Treytel-À Sugiez: histoire d'un complexe mégalithique néolithique, témoins d'habitats du Campaniforme et du Bronze ancien, Archéologie Neuchâteloise 47, Neuchâtel.

Gruet, M., 1967. Inventaire des Mégalithes de la France: Maine-etLoire. CNRS, $1^{\text {er }}$ suppl. Gallia Préhistoire, Paris.

Guibert, P., Lahaye C., Bechtel F., 2009. The importance of U-series disequilibrium of sediments in luminescence dating: A case study at the Roc de Marsal Cave (Dordogne, France), Radiation Measurements 44: 223-231.

Jubert, A., 1974. Contribution à l'étude des signes gravés des mégalithes armoricains: l'idole néolithique. Université de Nantes, mémoire de Maîtrise, France.

LASKARIS, N. et LiRITZIS, I., 2011. A new mathematical approximation of sunlight attenuation in rocks for surface luminescence dating, Journal of Luminescence 131: 1874-1884.

Le Roux, C.T, Lecerf, Y., Gautier M., 1989. Les mégalithes de Saint-Just (llle-et-Vilaine) et la fouille des alignements du Moulin de Cojou. Revue Archéologique de l'Ouest, 6: 5-29.

L'Helgouac'h, J., 1983. Les Idoles qu'on abat. Bulletin société polymatique du Morbihan, 110: 57-68.

L'Helgouac'H, J., Cassen, S., 2009. Les rapports d'interventions sur les trois campagnes de fouilles trisannuelles à la Table des Marchands (1986-1988/1989-1991/1992-1994). In S. CASSEN (dir.). Autour de la Table. Explorations archéologiques et discours savants sur des architectures néolithiques à Locmariaquer, Morbihan (Table des Marchands et Grand Menhir). Actes du colloque international, Vannes, Université de Bretagne-Sud, 5-7 octobre 2007. Université de Nantes, LARA: 35-140.

Liritzis, I., Murray, A.S. et Vafiadou, A., 2007. Optically stimulated luminescence (OSL) dating investigations of rock and underlying soil from three case studies. Journal of Archaeological Science 34: 1659-1669.

Liritzis, I., Polymeris, G., Zacharias, N., 2010. Surface luminescence dating of 'Dragon Houses' and Armena Gate at Styra (Euboea, Greece), Mediterranean Archaeology \& Archaeometry, Special Issue, vol. 10, no 3: 65-81.

Liritzis, I., Vafiadou, A., 2005. Dating by luminescence of ancient megalithic masonry, Mediterranean Archaeology \& Archaeometry 5, 1: 25-38.
LiRITZIS, I., 2011. Surface dating by luminescence: an overview, Geochronometria 38: 292-302.

López-Romero, E., 2011. Datación por luminiscencia ópticamente estimulada de monumentos megalíticos: contexto y perspectivas. Menga n ${ }^{\circ}$ 1: 193-214.

Meuret, J.-C., 2001. Prospection archéologique en milieu forestier. L'exemple de la haute Armorique, Revue archéologique de l'Ouest, suppl. 9: 307-315.

Мınoт, R., 1964. Sur les Gravures mégalithiques du Morbihan. Bulletin société polymathique du Morbihan: 89-98.

Murray, A.S, Wintle, A.G., 2000. Luminescence dating of quartz using an improved single-aliquot regenerative-dose protocol. Radiation Measurements 32: 57-73.

Parker Pearson, M., Marshall, P., Pollard, J., Richards, C., Thomas, J., Welham, K. à paraître. Stonehenge. In The Oxford Encyclopaedia of European Prehistory, Oxford.

Reimer P.J., Baillie M.G.L., Bard E., Bayliss A., Beck J.W., Blackwell P.G., Bronk Ramsey C., Buck C.E., Burr G.S., Edwards R.L., Friedrich M., Grootes P.M., Guilderson T.P., Hajdas I., Heaton T.J., Hogg A.G., Hughen K.A., Kaiser K.F., Kromer B., McCormac F.G., Manning S.W., Reimer R.W., Richards D.A., Southon J.R., Talamo S., Turney C.S.M., van der Plicht J., Weyhenmeyer C.E., 2009. IntCal09 and Marine09 Radiocarbon Age Calibration Curves, 0-50,000 Years cal BP, Radiocarbon, 51, n 4, 1111 1150 .

Smith, B.W., Rhodes, E.J., 1994. Charge movements in quartz and their relevance to optical dating, Radiation Measurements 23: 329-333.

Trautmann, F., 1988. Notice explicative carte géologique de la France (1/50000), feuille Nozay (420), BRGM Carte géologique par F. Trautmann, M. Donnot, D. Lemaire, M.P. Cagnet-Mawhin 1987, Orléans.

Tribolo, C., Mercier, N., Valladas, H., 2003. Attempt at using the single-aliquot regenerative-dose procedure for the determination of equivalent doses of Upper Palaeolithic burnt stones, Quaternary Sciences Review 22: 1251-1256.

Vieillevigne, E., Guibert, P., Zuccarello, A. R., Bechtel, F., 2006. The potential of optically stimulated luminescence for medieval building, a case study at Termez (Uzbekistan). Radiation Measurements 41: 991-994.

Wintle, A., Murray, A., 2006. A review of quartz optically stimulated luminescence characteristics and their relevance in single-aliquot regeneration dating protocols, Radiation Measurements 41: 369-391. 